\title{
EXTENDED HI STRUCTURES IN THE IRREGULAR GALAXY NGC 4449
}

\author{
HUGO VAN WOERDEN \\ Kapteyn Institute, Postbus 800, 9700 AV Groningen, The Netherlands \\ DEIDRE A. HUNTER \\ Lowell Observatory, $1400 \mathrm{~W}$ Mars Hill Road, Flagstaff AZ 86001, USA \\ AND \\ ERIC M. WILCOTS AND JAY S. GALLAGHER \\ Washburn Observatory, 475 N. Charter Street, Madison WI 53706, USA
}

NGC 4449 is a bright $(B=9.5)$, nearby $(4 \mathrm{Mpc})$ irregular galaxy with a high surface brightness and strong star-formation activity; for photos see Hunter and Gallagher (1989). Using the Effelsberg 100-meter dish, van Woerden et al. (1975) - and later also Bajaja et al. (1994) - found its HI distribution to extend, at the level $2 \times 10^{19}$ atoms cm${ }^{-2}$, over a full degree, or ten times the $D_{25}$ diameter. Van Woerden et al. also mentioned that the HI velocity gradient in this outer disk was opposite to that measured at Westerbork over the innermost 4 arcminutes.

With the VLA D-array, we have used a mosaic of $3 \times 3$ pointings to map the HI at resolutions of $1.0^{\prime}$ and $10 \mathrm{~km} \mathrm{~s}^{-1}$ over a field (FWHM) of $1^{\circ}$ diameter (Hunter et al. 1998). The HI distribution (Fig. 1) shows the following features: 1) a bright inner body, centred on the optical galaxy and dominated by an annulus of $5 \mathrm{kpc}$ diameter; 2) a symmetric elliptical - probably highly inclined - disk of $35 \times 15 \mathrm{kpc}$ diameter, with strong condensations at its NE and SW ends; 3) a set of narrow streamers, consisting of straight segments making abrupt angles, and containing several bright clouds. The HI mass in the inner body and elliptical disk together is $12 \times 10^{8} \mathrm{M}_{\odot}$; the streamers (about $7 \times 10^{8} \mathrm{M}_{\odot}$ of $\mathrm{HI}$ ) are surrounded by a diffuse HI distribution, missed by the VLA interferometers but detected by the Effelsberg single-dish.

While the kinematics inside $4 \mathrm{kpc}$ radius is complex, the elliptical disk outside $8 \mathrm{kpc}$ radius appears to rotate at about $75 \mathrm{~km} \mathrm{~s}^{-1}$, in a period of order $1 \mathrm{Gyr}$; its velocity gradient (increasing from NE to SW) is confirmed to be opposite to that in the inner body. The velocity field of the streamers is highly ordered; the velocity difference of $160 \mathrm{~km} \mathrm{~s}^{-1}$ (South - North), if interpreted as rotation, would imply a total mass of $10^{11} \mathrm{M}_{\odot}$ in the system; however, it is not clear that the streamers actually rotate around NGC 4449 . With $100 \mathrm{~km} \mathrm{~s}^{-1}$ velocity. the travel time along the filament from the SW corner to the NE end would be about $1 \mathrm{Gyr}$. Velocity dispersions are about $10 \mathrm{~km} \mathrm{~s}^{-1}$ in the brighter parts of disk and streamers; the Northern joint and the SW crossing have broader or complex profiles. The time scale for expansion of the streamers is of order $200 \mathrm{Myr}-$ unless self-gravity keeps them together.

What caused these peculiar structures? Even if the extended distribution found at Effelsberg represents delayed accumulation of primordial gas, we think the complex set of streamers must be due to external, tidal perturbations. The HI distribution is strikingly similar to that in the M81-M82-NGC3077 triplet (van der Hulst 1979, Yun et al. 1994), and three bodies may indeed $b \in$ required to explain the sharp corners. The analogon of NGC 3077, at a closely corresponding position, may be provided by the gas cloud (a failed galaxy?) at the SW corner, where two filaments cross. But where is the third body? We consider several possibilities.

The nearby dwarf-irregular DDO 125 , just $37^{\prime}$ SSW of NGC 4449 , appears undisturbed and has fairly normal global properties, including $1 \times 10^{8} \mathrm{M}_{\odot}$ of $\mathrm{HI}$ and $M(\mathrm{HI}) / L_{\mathrm{B}}=0.4$. Could it once have had 8 times more gas and twice as many stars, and lost most of its gas and half of its stars over a period of $10^{9}$ years, without looking disturbed? If spread over an area of $100 \times 5 \mathrm{kpc}^{2}$, the stars would produce an almost-undetectable surface brightness of only $28 \mathrm{mag} \operatorname{arcsec}^{-2}$. Other, major possible perturbers are at distances exceeding $4^{\circ}=300 \mathrm{kpc}$, implying timescales of order 3 Gyr, possibly too long compared to that of the streamers. 


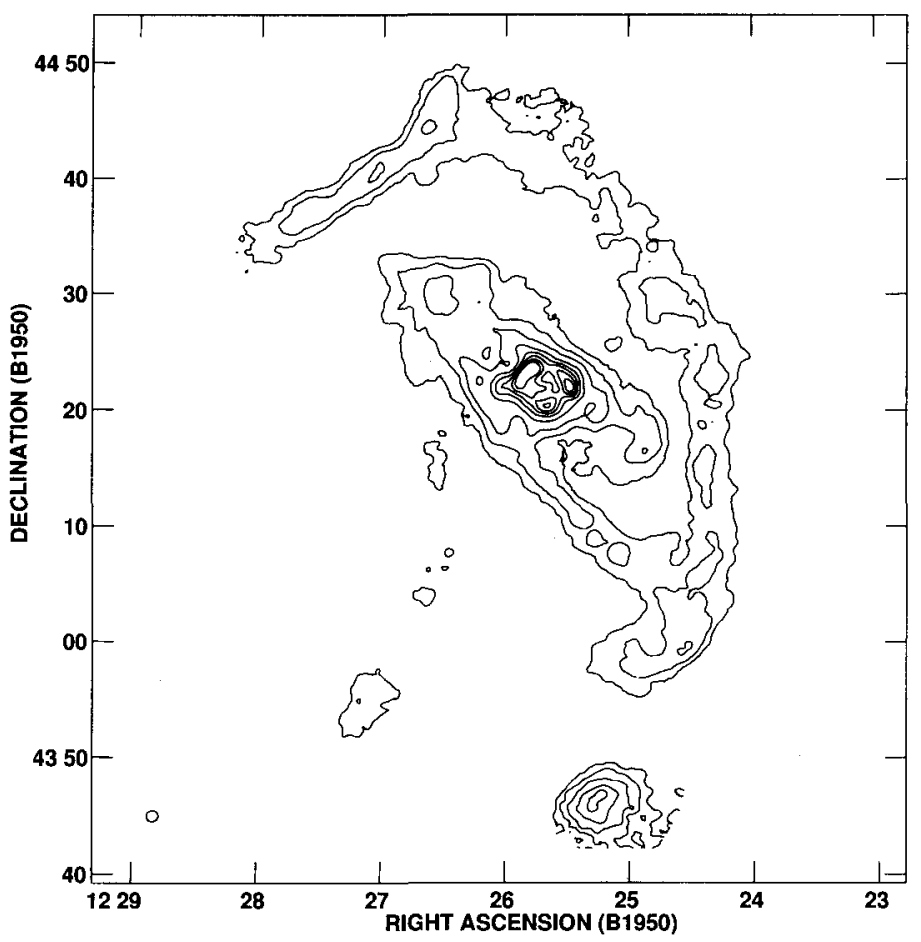

Figure 1. Distribution of HI column density; contour levels are 1, 2.5, 5, 10, 15, 20, 25 and 30 times $0.68 \times 10^{20}$ atoms $\mathrm{cm}^{-2}$. The ellipse at lower left is the half-maximum contour of the synthesized beam.

Or has the third body been swallowed by NGC 4449? Such a merger might have caused its current strong star formation, the counter-rotation of the inner HI, and its irregular distribution. The streamer running from the inner disk to the SW corner might then be a Toomire \& Toomre (1972)-type bridge. Possible clues may come from measurement of the rotation of the inner stellar galaxy, from age analysis of its star clusters, and from a detailed study of the inner HI body and its possible interaction with the other subsystems.

\section{Acknowledgements}

The VLA is part of the U.S. National Radio Astronomy Observatory, operated by Associated Universities, Inc., under contract with the National Science Foundation. HvW thanks the Leids Kerkhoven-Bosscha Fonds and Washburn Observatory for support of his visit to Madison and to the IAU Assembly. DAH and EMW acknowledge support by NSF under Grants No. AST-9616940 and 9616907 .

\section{References}

Bajaja, E., Huchtmeier, W.K., \& Klein, U. 1994, A\&A, 285, 388

Hunter, D.A., \& Gallagher, J.S. 1989, Science, 243, 1557

Hunter, D.A., Wilcots, E.M., van Woerden, H., Gallagher, J.S., \& Kohle, S. 1998, ApJ, in preparation

Toomre, A., \& Toomre, J. 1972, ApJ, 178, 623

van der Hulst, J.M. 1979, A\&A, 75, 97

van Woerden, H., Mebold, U., \& Bosma, A. 1975, in La Dynamique des Galaxies Spirales, ed. Weliachew, L., Paris:CNRS, p. 483

Yun, M.S., Ho, P.T.P., \& Lo, K.Y. 1994, Nature, 372, 530 